\title{
Imaging Characteristics of Wingless Pathway Subgroup Medulloblastomas: Results from the German HIT/SIOP-Trial Cohort
}

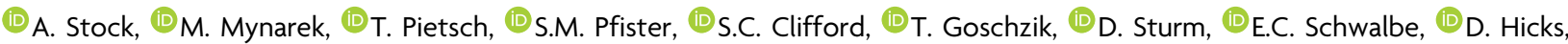 \\ (1) S. Rutkowski, ${ }^{\circ}$ B. Bison, ${ }^{-}$M. Pham, and ${ }^{\circ}$ M. Warmuth-Metz
}

\begin{abstract}
BACKGROUND AND PURPOSE: In addition to the 4 histopathologically defined entities of medulloblastoma, 4 distinct genetically defined subgroups have been included in the World Health Organization classification of 2016. The smallest subgroup is the medulloblastoma with activated wingless pathway. The goal of this study was to identify a typical MR imaging morphology in a larger number of pediatric patients with wingless pathway medulloblastoma.
\end{abstract}

MATERIALS AND METHODS: From January 2001 to October 2017, of 75 patients with histologically confirmed and molecularly subgrouped wingless pathway medulloblastomas recruited to the German Pediatric Brain Tumor (HIT) trials, 38 patients (median age, $12.8 \pm 4.6$ years at diagnosis; 24 [63.2\%] female) had preoperative imaging that passed the entry criteria for this study. Images were rated by the local standardized imaging criteria of the National Reference Center of Neuroradiology. Additionally, a modified laterality score was used to determine tumor localization and extension.

RESULTS: Twenty-eight of 38 (73.7\%) were primary midline tumors but with a lateral tendency in $39.3 \%$. One extensively eccentric midline tumor was rated by the laterality score as in an off-midline position. Five tumors were found in the cerebellopontine angle; 3 , in the deep white matter; and 2, in a cerebellar hemisphere. Leptomeningeal dissemination was rare (11.5\%). In $60.5 \%$, intratumoral blood-degradation products were found, and $26.3 \%$ showed cysts with blood contents.

CONCLUSIONS: According to our observations, wingless pathway medulloblastomas are not preferentially off-midline tumors as postulated in previous studies with smaller wingless pathway medulloblastoma cohorts. Dense intratumoral blood-degradation products and cysts with blood contents are frequently found and might help to differentiate wingless pathway medulloblastoma from other medulloblastoma subtypes.

ABBREVIATIONS: CMB = classic medulloblastoma; $\mathrm{CPA}=$ cerebellopontine angle; HIT = German abbreviation for brain tumor; LCMB = large-cell medulloblastoma (according to the World Health Organization classification of 2007); LS = laterality score; MB = medulloblastoma; SHH = sonic hedgehog pathway; WHO = World Health Organization; WNT $=$ wingless-activated; WNT-MB $=$ WNT-activated medulloblastoma

A ccording to the revised WHO Classification of Tumors of the Central Nervous System of 2016, ${ }^{1}$ medulloblastoma (MB) is

Received May 14, 2019; accepted after revision September 3.

From the Department of Neuroradiology (A.S., B.B., M.P., M.W.-M.), University Hospital Wuerzburg, Wuerzburg, Germany; Department of Pediatric Hematology and Oncology (M.M., S.R.), University Medical Center Hamburg-Eppendorf, Hamburg, Germany; Institute of Neuropathology (T.P., T.G.), DGNN Brain Tumor Reference Center, University of Bonn Medical Center, Bonn, Germany;

Department of Pediatric Hematology and Oncology (S.M.P.), Heidelberg University Hospital, Heidelberg, Germany; Division of Pediatric Neurooncology (S.M.P.), German Cancer Consortium, German Cancer Research Center, Heidelberg, Germany; Hopp Children's Cancer Heidelberg (S.M.P., D.S.), Heidelberg, Germany; Wolfson Childhood Cancer Research Centre (S.C.C., E.C.S., D.H.), Northern Institute for Cancer Research, Newcastle University, Newcastle upon Tyne, UK; and Department of Applied Sciences (E.C.S.), Northumbria University, Newcastle upon Tyne, UK.

This study was supported by grants from the German Children's Cancer Foundation and Cancer Research UK.

Paper previously presented, in part, as a scientific abstract at: Annual Meeting of the German Society of Neuroradiology, October 10, 2018; Frankfurt, Germany. not considered a single tumor entity but represents several entities with different cells of origin, location, biology, genetic or (epi-)genetic alterations, histology, and clinical behavior. According to the concept of an integrated diagnosis in the World Health Organization (WHO) classification of 2016, medulloblastoma (MB) entities are defined by both histologic and molecular/ genetic features, allowing a precise assignment of patients for risk-adapted stratification in current therapeutic studies and the comparison with results of study cohorts in the past. All $\mathrm{MB}$ entities correspond to WHO grade IV. For a definition of the histologic diagnosis, the tumors should be assigned to 1 of the 4 entities: classic (CMB), desmoplastic nodular, extensive

Please address correspondence to Annika Stock, MD, Department of Neuroradiology, University Hospital Wuerzburg, Josef-Schneider-Str 11, D-97080 Wuerzburg, Germany; e-mail: stock_a@ukw.de

http://dx.doi.org/10.3174/ajnr.A6286 

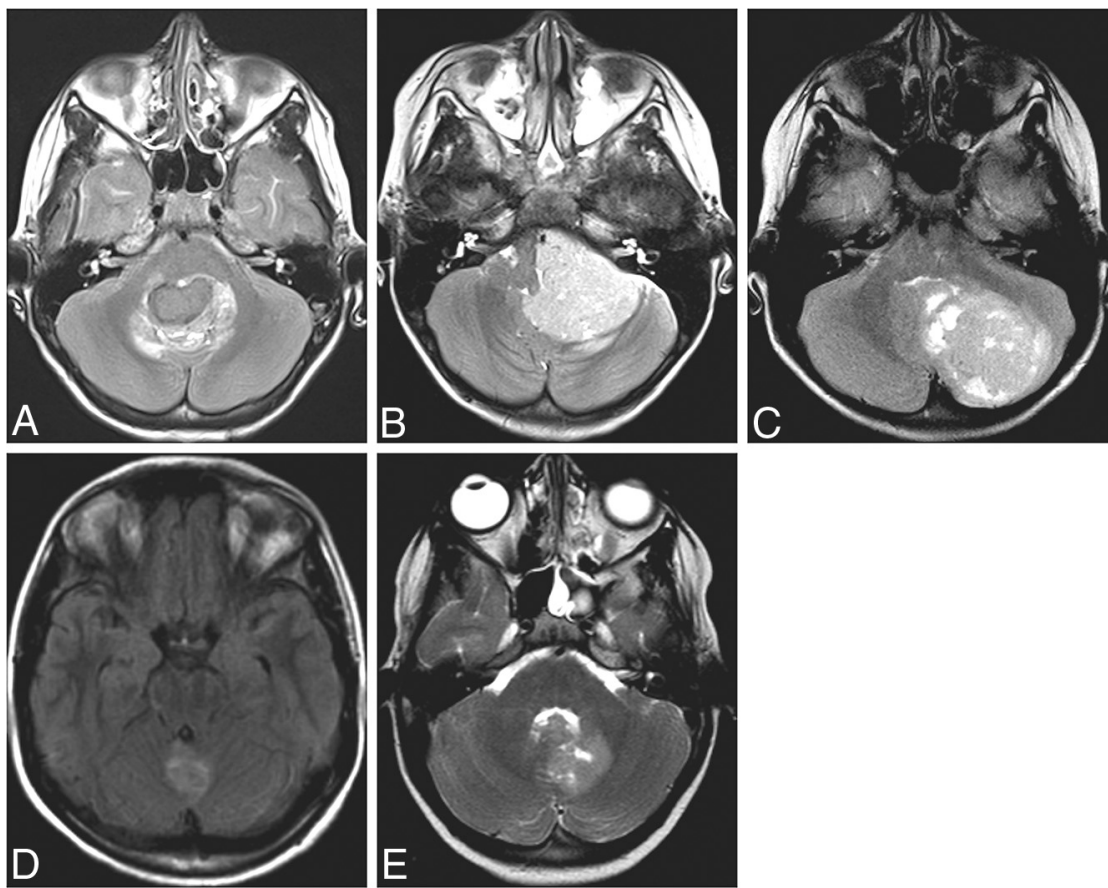

FIG 1. $A-C$, and $E$, T2WI. D, T2-FLAIR. Examples for primary tumor positions in WNT-MB. The midline fourth ventricle $(A)$, cerebellopontine angle $(B)$, cerebellar hemisphere $(C)$, midline cerebellar vermis $(D)$, and periventricular deep white matter $(E)$.

nodularity, or large cell/anaplastic MB. Large-cell MB (LCMB) and anaplastic $\mathrm{MB}$ were separate histologic entities before $2016{ }^{2}$ In addition to these histologic entities, 4 genetically defined subgroups have been introduced by the WHO classification of 2016. ${ }^{1}$ Two subgroups are defined by their genetic pathway, wingless-activated (WNT) and sonic hedgehog-activated $(\mathrm{SHH}) \mathrm{MB}$, the latter with or without an accompanying TP53 mutation. The non-WNT/non-SHH subgroup is provisionally subclassified into group 3 and 4 medulloblastomas, which seem to be overlapping variants. WNT-MBs are characterized by activating mutations of CTNNB $1,{ }^{3,4}$ which encodes a central component of the WNT pathway or, alternatively, by inactivating mutations of $A P C,{ }^{3,5} A X I N 11^{6,7}$ or $A X I N 2,{ }^{8}$ leading to nuclear accumulation of $\beta$-catenin. Accounting for approximately $10 \%$ of all MBs, WNT-MBs form a small-but-distinct MB entity. In children, they show a significantly better progression-free survival and overall survival compared with other subgroups. ${ }^{4,9-11}$ In patients with WNT-MB younger than 16 years of age at diagnosis, a progression-free survival of $100 \%$ for 5 years has been observed. ${ }^{11}$

Current therapy studies, such as the SIOP-PNET5 (NCT02066220) medulloblastoma trial, have implemented upfront genetic evaluations so that patients with low-risk WNTMBs are eligible for radiation therapy reduction aimed at reducing late effects, with the therapy increasingly being adapted to the specific risk profile of each patient. Additionally, radiologic imaging signatures have become apparent for certain brain tumors and their underlying genetic profiles. The term "radiomics" is increasingly applied to describe the association between imaging phenotype and tumor genotype. A priori knowledge of the radiologic imaging phenotype can become clinically meaningful, for example, when it offers the neurosurgeons and neurooncologists a better preoperative estimation of the prognosis and risk of relapse of the tumor. Based on the principle of radiomics, our aim was to evaluate specific imaging characteristics of WNT-MB. Due to the large number of participating study sites, automated techniques of radiomics image analyses including algorithms of machine learning, were not included in this study.

Besides standard imaging characteristics, radiologic studies have mainly focused on correlations of tumor position and genetic information. A first radiologic subgroup analysis showed a preferential tumor location for WNT-MB in the cerebellopontine angle (CPA) and for sonic hedgehog-activated medulloblastoma (SHH-MB) in the cerebellar hemisphere. ${ }^{12}$ More recent studies with sample sizes of WNT-MB of $n=15,{ }^{13} n=16,{ }^{14}$ and $n=17^{15}$ yielded heterogeneous results with regard to tumor localization. The goal of the present study was to analyze structural MR imaging features according to defined MR imaging criteria in a large number of genetically determined WNT-MBs $(n=38)$ in children within the framework of the German Pediatric Brain Tumor (HIT) trials and the National Reference Center for Neuroradiology.

\section{MATERIAL AND METHODS Study Cohort}

Cases were retrospectively collected from the data base of the National Reference Center for Neuroradiology (Department for Neuroradiology, Wuerzburg University Hospital) for the German Brain Tumor (HIT) trials, conducted in Germanspeaking countries of Europe. All patients were registered to the HIT-2000 trial (January 2001 to December 2011 [NCT00303810]), including participants of the German PNET 4 cohort from January 2001 to December 2007 [NCT01351870]), the HIT-2000 interim registry (recruiting January 2012 to December 2014 [NCT02238899]), the I-HITMED registry (recruiting since January 2015 [NCT02417324]), or the SIOP-PNET 5 MB trial (recruiting since June 2014 [NCT02066220]). Each patient or legal guardian signed an informed consent declaration when entering the study allowing the scientific evaluation of biologic and imaging data. All cases were centrally reviewed at the National Brain Tumor Reference Center of the German Society of Neuropathology and Neuroanatomy, Institute of Neuropathology, Bonn University. Patients were eligible if they had histopathologic and genetic classification data and preoperative cranial and spinal imaging 
Table 1: Overview of absolute and relative (\%) frequencies of MR imaging features

\begin{tabular}{|c|c|c|c|}
\hline & Available Data & No. & $\%$ \\
\hline Sex & 38 & & \\
\hline Male & & 14 & 36.8 \\
\hline Female & & 24 & 63.2 \\
\hline Histology & 38 & & \\
\hline $\mathrm{CMB}$ & & 36 & 94.7 \\
\hline $\mathrm{AMB}^{\mathrm{a}}$ & & 1 & 2.6 \\
\hline $\mathrm{LCMB}^{\mathrm{a}}$ & & 1 & 2.6 \\
\hline Hydrocephalus & 38 & & \\
\hline No & & 15 & 39.5 \\
\hline Slight & & 6 & 15.8 \\
\hline Moderate & & 15 & 39.5 \\
\hline Severe & & 2 & 5.3 \\
\hline Enhancement & 38 & & \\
\hline No & & 0 & 0 \\
\hline Light & & 4 & 10.5 \\
\hline Moderate & & 12 & 31.6 \\
\hline Strong & & 22 & 57.9 \\
\hline Enhancing area & 38 & & \\
\hline $0 \%-25 \%$ & & 0 & 0 \\
\hline $26 \%-50 \%$ & & 1 & 2.6 \\
\hline $51 \%-75 \%$ & & 1 & 2.6 \\
\hline $76 \%-100 \%$ & & 36 & 94.7 \\
\hline T2-weighted images & 37 & & \\
\hline Homogeneous & & 6 & 16.2 \\
\hline Inhomogeneous & & 31 & 83.8 \\
\hline Hyperintense & & 4 & 10.8 \\
\hline Isointense & & 20 & 54.1 \\
\hline Hypointense & & 13 & 35.1 \\
\hline T1-weighted images & 36 & & \\
\hline Homogeneous & & 15 & 41.7 \\
\hline Inhomogeneous & & 21 & 58.3 \\
\hline Hyperintense & & 1 & 2.8 \\
\hline Isointense & & 8 & 22.2 \\
\hline Hypointense & & 27 & 75 \\
\hline Cysts & 38 & & \\
\hline No & & 20 & 52.6 \\
\hline As bright as the CSF & & 3 & 7.9 \\
\hline Brighter than the CSF & & 5 & 13.2 \\
\hline Blood-fluid level & & 10 & 26.3 \\
\hline $\begin{array}{l}\text { Blood-degradation products on } \\
\text { TIWI, T2WI or } T 2^{*} / \mathrm{SWI}\end{array}$ & 38 & & \\
\hline Yes & & 23 & 60.5 \\
\hline No & & 15 & 39.5 \\
\hline Mass of iron contents on $\mathrm{T} 2^{*} / \mathrm{SWI}$ & 18 & & \\
\hline No & & 2 & 11.1 \\
\hline$<50 \%$ & & 11 & 61.1 \\
\hline$>50 \%$ & & 5 & 27.8 \\
\hline Dissemination & 35 & & \\
\hline No & & 31 & 88.6 \\
\hline M2 & & 1 & 2.9 \\
\hline M3 & & 1 & 2.9 \\
\hline $\mathrm{M} 2+3$ & & 2 & 5.7 \\
\hline
\end{tabular}

Note:-AMB indicates anaplastic medulloblastoma according to the WHO classification of 2007

a According to the WHO classification of 2007.

data. Images on $\mathrm{x}$-ray films and examinations with strong movement artifacts were excluded.

\section{Molecular Analysis}

Histological diagnosis was made at inclusion into the trial according to the WHO classification valid at the time. Patients were diagnosed by a combination of histologic examination by immunohistochemistry and Sanger sequencing of exon 3 of CTNNB1. ${ }^{16,17}$ In case of negative CTNNB1 mutation, APC, AXIN1, and AXIN2 sequences were assessed additionally. ${ }^{11}$ Neuropathologic evaluation and CTNNB1 mutation analysis were supplemented by $450 \mathrm{k}$ DNA methylation microarray (Illumina, San Diego, California) if applicable. ${ }^{18,19}$ Where tissue was too scarce for analysis by methylation microarray, subgroup was assigned using the mass spectrometry-minimal methylation classifier assay in addition to immunohistochemistry and CTNNB1 mutation analysis. ${ }^{11,20}$ Where the initial work-up did not include prospective assessment of WNT activation, this was done during retrospective work-up as previously described. ${ }^{11,18}$ Patients were considered to have WNT-MB if molecular analysis confirmed a mutation in CTNNB1 or APC and/or assignment to the WNT-subgroup by methylation profiling or mass spectrometry-minimal methylation classifier.

\section{Imaging Analysis}

All MR imaging datasets were assessed in consensus by 2 neuroradiologists dedicated to pediatric brain tumor imaging (M.W.-M. and A.S.).

Multicenter data acquisition resulted in nonuniform MR imaging protocols, sequence technique, parameters, and field strength. Inclusion was possible with T2WI or T2-FLAIR and contrast-enhanced T1WI. The primary tumor location was determined as: cerebellar hemisphere, deep white matter, CPA, cerebellar vermis, or fourth ventricle (Fig 1). Deep white matter, cerebellar hemisphere, and CPA were rated as primary lateral positions. When the CPA was involved, the status of the fourth ventricle and the foramen of magendie was recorded additionally. Fourth ventricle and cerebellar vermis were defined as primary midline positions. Additionally, laterality of primary midline tumors was assessed according to a modification of the laterality score (LS) by Patay et al. ${ }^{14}$ Primary positions in the fourth ventricle or the cerebellar vermis were rated as midline (LS-0). Fourth ventricle or cerebellar vermis plus bilateral recess extension was rated as LS-0 as well. Midline tumors were rated as moderately lateralized in case of tumor extension into only the unilateral recess or bilateral recesses plus 1 CPA (LS-1). Extension into only the unilateral recess and further into the ipsilateral CPA was rated as heavily lateralized and off-midline (L-2).

Local standardized diagnostic parameters were supplemented and used for the imaging assessment. The tumor volume is calculated using the approximation of the ellipsoid volume formula $\mathrm{A} \times \mathrm{B} \times \mathrm{C} \frac{1}{2}$, where $\mathrm{A}, \mathrm{B}$, and $\mathrm{C}$ are the maximum dimensions in the standard anterior-posterior, craniocaudal, and transverse planes. The largest diameter of the perifocal edema was measured in centimeters. Signal intensity and homogeneity of the tumor were assessed in comparison with gray matter on T2WI and T1WI without contrast enhancement. Intensity and percentage of enhancing volume after gadolinium application were estimated. The contrast-enhancing area of the tumor mass is mainly diffuse, whereas the rating was subjective in approximate percentages $(0 \%-25 \%, 26 \%-50 \%, 51 \%-75 \%, 76 \%-100 \%)$.

Hydrocephalus was rated as slight, moderate, and severe (slight meaning only visible dilation of the ventricles, moderate showing CSF pressure caps, and severe showing pressure caps 
Table 2: Overview of the tumor proposed epicenter and direction of extension

\begin{tabular}{|c|c|c|c|c|c|}
\hline $\begin{array}{l}\text { Primary Location } \\
\text { (No. of WNT-MBs) }\end{array}$ & Extension & & Further Extension & & LS \\
\hline \multirow[t]{6}{*}{ Fourth ventricle (25) (66\%) } & Unilateral recess & $n=7$ & Plus uni CPA & $n=1$ & 2 \\
\hline & & & No & $n=6$ & 1 \\
\hline & Bilateral recesses & $n=16$ & Plus uni CPA & $n=4$ & 1 \\
\hline & & & Plus bi CPA & $n=0$ & 0 \\
\hline & & & No & $n=12$ & 0 \\
\hline & No extension & $n=2$ & & & 0 \\
\hline \multirow[t]{4}{*}{ Cerebellar vermis (3) (8\%) } & Unilateral recess & $n=1$ & & & 1 \\
\hline & Bilateral recesses & $n=1$ & & & 0 \\
\hline & $\begin{array}{l}\text { Fourth ventricle } \\
\text { only }\end{array}$ & $n=1$ & & & 0 \\
\hline & No extension & $n=0$ & & & 0 \\
\hline \multirow[t]{3}{*}{ CPA (5) (13\%) } & Unilateral recess & $n=4$ & $\begin{array}{l}\text { Plus fourth } \\
\text { ventricle }\end{array}$ & $n=4$ & 2 \\
\hline & Bilateral recesses & $n=0$ & & & 2 \\
\hline & No extension & $n=1$ & & & 2 \\
\hline \multirow[t]{4}{*}{ Deep WM (3) (8\%) } & Unilateral recess & $n=1$ & Plus uni CPA & $n=1$ & 2 \\
\hline & Bilateral recesses & $n=0$ & & & 2 \\
\hline & $\begin{array}{l}\text { Fourth ventricle } \\
\text { only }\end{array}$ & $n=1$ & & & 2 \\
\hline & No extension & $n=1$ & & & \\
\hline \multirow{4}{*}{$\begin{array}{l}\text { Cerebellar hemisphere (2) } \\
(5 \%)\end{array}$} & Unilateral recess & $n=2$ & Plus uni CPA & $n=1$ & 2 \\
\hline & Bilateral recesses & $n=0$ & & & 2 \\
\hline & $\begin{array}{l}\text { Fourth ventricle } \\
\text { only }\end{array}$ & $n=0$ & & & 2 \\
\hline & No extension & $n=0$ & & & 2 \\
\hline
\end{tabular}

Note:-uni indicates unilateral; bi, bilateral.

with compression of the sulci at the vertex). Fluid of intratumoral cysts was compared with the signal of CSF and defined as: as bright as CSF and brighter than CSF, presence of blood-degradation products like methemoglobin or hemosiderin was noted. Leptomeningeal dissemination was assessed only by MR imaging according to the classification of Chang et al. ${ }^{21}$ Cranial dissemination was rated as M2; spinal dissemination, as M3; and cranialplus-spinal dissemination, as $\mathrm{M} 2+3$. In addition to these standard diagnostic parameters, we rated the presence of blooddegradation products like methemoglobin and hemosiderin within the solid tumor mass as: no blood-degradation products, blood-degradation products in $<50 \%$ of the tumor volume, and blood-degradation products in $>50 \%$ of the tumor mass.

\section{RESULTS}

From January 2001 to October 2017, WNT activation was confirmed in 75 patients with MB. Preoperative cranial MR imaging and pre- or postoperative spinal MR imaging that passed entry criteria for this study were available in 38 patients with WNTMB. In this MR imaging cohort, age ranged from 5 to 21.6 years (median, $12.8 \pm 4.6$ years) and a female predominance (1.71:1, female/male ratio) was found. According to traditional histopathologic criteria, most WNT-MB cases of this cohort were CMB (36/38; 94.7\%) and further 2 large-cell/anaplastic MB (1 anaplastic MB and 1 LCMB diagnosed before 2016 [2.6\% each] according to the WHO classification of 2007). Thirty-four tumors were diagnosed by a combination of immunohistochemistry and Sanger sequencing of exon 3 of CTNNB1, each with a detectable mutation of CTNNB1. In 15 of these patients, 450k methylation microarray was supplemented and classified all tumors as WNT. There were 2 cases without Sanger sequencing: One was categorized as WNTactivated by mass spectrometry-minimal methylation classifier assay and the other one by $450 \mathrm{k}$ methylation microarray. In 1 tumor, analysis was negative for the CTNNB1 mutation but was classified as a WNT subgroup by $450 \mathrm{k}$ methylation microarray analysis, and additionally, a copy-neutral loss of heterozygosity within the chromosome arm 5q (APC) and a R213* mutation in the APC gene were identified. One patient was diagnosed as having WNT-MB based on tissue of tumor recurrence; here, CTNNB1 mutation and WNT-activation in $450 \mathrm{k}$ methylation microarray were detectable.

An overview of the MR imaging features is shown in Table 1, and primary tumor localization and extension are presented in Table 2. Twenty-eight tumors were primarily located in the midline position. Fourteen of 25 (56\%) WNT-MBs in the fourth ventricle and 2 of 3 in the cerebellar vermis were midline tumors without lateral tendency (LS-0). Eleven of 28 (39.3\%) WNT-MBs showed extension into 1 recess only or into both recesses with further extension into only $1 \mathrm{CPA}$ (LS-1). Only 1 WNT-MB located in the fourth ventricle extended into a unilateral recess and further into the ipsilateral CPA (LS2). In summary, $74 \%$ of all WNT-MBs showed primarily a midline position, but only $42 \%$ represented "pure" midline tumors, with nearly half of the midline tumors showing a lateral tendency (39.3\%). Only 1 (3.6\%) primarily midline-located WNT-MB was rated as off-midline according to our LS score because of its strong eccentric position. Five of 38 (13\%) WNT-MBs were positioned in the cerebellopontine angle; 2 of 38 (5\%), in the cerebellar hemisphere; and 3 of 38 (8\%), in the deep white matter. These positions were rated as off-midline. One CPA tumor showed some extension into the fourth ventricle. In both cerebellar hemisphere tumors, the ipsilateral recess was involved. One tumor with its epicenter in the deep white matter expanded into the fourth ventricle; and one, into the ipsilateral recess. In all deep white matter and cerebellar hemisphere tumors, WNT activation was confirmed by 450k DNA methylation microarray and a CTNNB1 mutation was found as well.

Most WNT-MBs were moderately (39.5\%) to very sharply (42.1\%) delineated. Eighteen of 38 (47.4\%) WNT-MBs contained cysts. In 10 of 18 (55.6\%) partly cystic tumors, the cysts contained blood-degradation products, visible as blood-fluid levels (Fig 2). The cyst contents were brighter than CSF in $27.8 \%$ and similar to CSF in $16.7 \%$. Twenty-three patients had hydrocephalus. Six (15.8\%) patients showed slight, and 15 (39.5\%) patients, moderate hydrocephalus; only 2 patients $(5.3 \%)$ had severe 

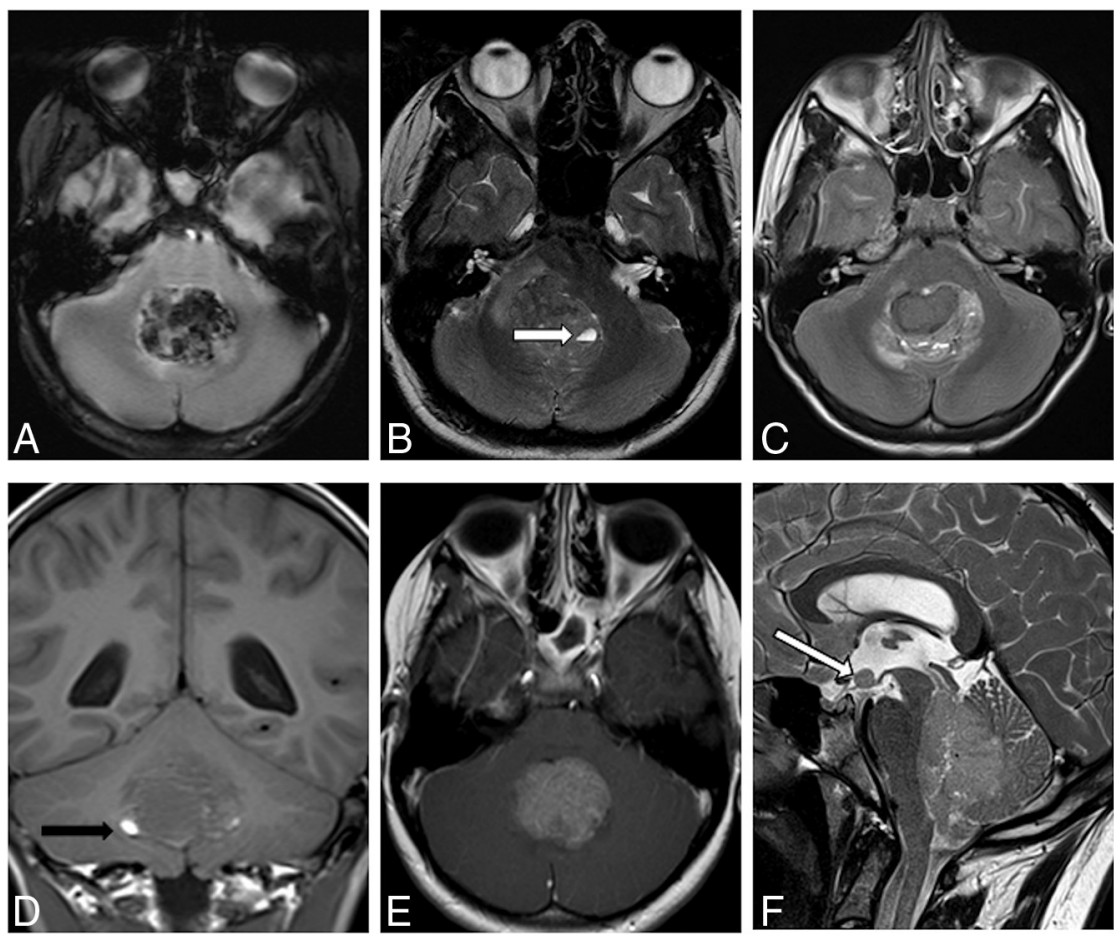

FIG 2. A, Deoxygenated blood in T2*WI. B, Intratumoral cyst with a blood-fluid level in T2WI $(\rightarrow)$. C, Inhomogeneous signal in T2WI. D, Hypointense signal in TIWI and small areas of methemoglobin $(\mathrm{eg}, \rightarrow$ ). E, One hundred percent contrast enhancement in TIWI. F, Leptomeningeal dissemination in T2WI, M2 on the floor of the third ventricle $(\rightarrow)$.

hydrocephalus. Fifteen (39.5\%) patients had no hydrocephalus at diagnosis. Tumor signal intensity in T2WI compared with the supratentorial cortex was predominantly isointense $(54.1 \%)$ to hypointense (35.1\%). Only 4 tumors (10.8\%) were hyperintense in comparison with the signal intensity of the cortex on T2WI. In 1 child, no standard T2 sequence but a FLAIR sequence was available for evaluation. Thirty-one (83.8\%) tumors showed inhomogeneous signal on T2WI; only 6 of 37 (16.2\%) showed homogeneous signal. On T1WI, the signal intensity was hypointense in 27 of 38 (75\%) tumors. Eight (22.2\%) tumors showed an isointense signal, and only 1 tumor (2.8\%) showed a completely hyperintense signal. Two patients had no standard unenhanced T1WI at diagnosis. Signal on T1WI was homogeneous in $41.7 \%$ and inhomogeneous in $58.3 \%$. Thirty-six of 38 (94.7\%) WNT-MBs showed contrast enhancement in $76 \%-100 \%$ of the tumor volume; only $1 \mathrm{WNT}$ MB showed an enhancing volume of $26 \%-50 \%$, and 1 WNT-MB, $26 \%-50 \%$. Signs of bleeding in T1WI, T2WI, or susceptibilityweighted images were found in 23/38 (60.5\%).

In 18 of those cases, susceptibility-weighted images such as SWI or T2* sequences had been acquired. Sixteen of 18 (88.9\%) susceptibility-weighted images showed iron content. Five of 16 MBs showed iron deposition in $>50 \%$ of the tumor mass. The extension of perifocal edema ranged from 0 to $2.6 \mathrm{~cm}$ (mean, $0.66 \pm 0.62 \mathrm{~cm})$. Twenty-seven of $38(71.1 \%) \mathrm{MBs}$ showed perifocal edema. Tumor volume ranged from 2.68 to $68.59 \mathrm{~cm}^{3}$ (mean, $27.79 \pm 16.87 \mathrm{~cm}^{3}$ ). LCMBs are usually significantly smaller than other histologic MB types (mean, $15 \mathrm{~cm}^{3}$ ) as described previously. ${ }^{22}$ By means of this value from the literature as a cutoff between large and small tumor sizes, 10 of $40 \mathrm{WNT}$ tumors were small (range, 2.68$12.77 \mathrm{~cm}^{3}$ ); all of them presented with classic histology. The 2 large-cell/anaplastic MBs had volumes of 29.81 and $46.39 \mathrm{~cm}^{3}$. Leptomeningeal dissemination at diagnosis was assessable in 35 patients and was positive in $11.5 \%$ (4 of 35). One patient showed cranial dissemination (M2), 2 had cranial and spinal dissemination $(\mathrm{M} 2+3)$, and 1 patient showed isolated spinal dissemination (M3). All 4 disseminated WNT-MBs showed classic histology in the primary tumor.

\section{DISCUSSION}

To our knowledge, this series represents the largest cohort of WNT-MBs analyzed for characteristic MR imaging features thus far. Our results show that the preferred primary position of our WNT-MBs is the midline fourth ventricle or vermis in 28 of 38 (74\%). Perreault et al ${ }^{12}$ were the first to report that WNT-MBs are characterized by an off-midline position in $75 \%$ ( 3 of 4 were located in the CPA or cerebellar peduncle), leading to a positive predictive value of $100 \%$ for this tumor site. In our cohort, only 5 of 38 (13\%) WNT-MBs had their epicenter in the CPA; altogether, only $26 \%$ were primarily located in an off-midline position. This is much lower compared with earlier study results. ${ }^{12-14}$ Gibson et $\mathrm{al}^{23}$ indicated that WNT-MBs arise in the lower rhombic limb from progenitor cells of nuclei in the dorsal brain stem and all 6 WNT-MBs in this publication were reported as midline tumors. Similarly, Eastowska et $\mathrm{al}^{24}$ found $5 / 6$ and Teo et $\mathrm{al}^{25}$ described 5 WNTMBs in a midline position. On the basis of the hypothesis of Gibson et $\mathrm{al}^{23}$ on the individual tumor origin, the pathways of extension were evaluated in more detail. With a self-defined score, Patay et $\mathrm{al}^{14}$ emphasized the lateralized position of WNTMBs (50\% off-midline position). We support the conclusion of Patay et al that WNT-MBs are paramedian midline tumors, describing a lateralized position in 22/38 tumors in our cohort. However, a purely CPA localization (5 of 38 ) seems to be much rarer than previously noted. ${ }^{12}$ The primary midline position is in line with the hypothesis that WNT-MBs arise from the dorsal brain stem and grow within the CSF spaces. Notably, the fourth ventricle may be the preferred pathway of tumor growth because it offers the least resistance. With only 3 tumors having their epicenter in the deep white matter surrounding the fourth ventricle in our study, this localization was significantly less frequent compared with that in previous studies ( $8 \%$ versus $20 \%$ ). ${ }^{13}$

While Perreault et $\mathrm{al}^{12}$ and Mata-Mbemba et $\mathrm{al}^{13}$ assigned a cerebellar hemispheric origin as highly predictive of $\mathrm{SHH}$-activated $\mathrm{MB}$, we found 2 WNT tumors located in the cerebellar 
hemisphere. However, both tumors were large, rendering the definition of their origin in differentiation between hemisphere and paraventricular white matter to be challenging. These different tumor locations in our cohort and partially divergent results compared with the previous studies question whether the offmidline or midline position has any useful diagnostic predictive value. The 4 genetic subgroup model was refined by Taylor et $\mathrm{al}^{26}$ in 2012 and has been updated recently. ${ }^{27-29}$ Further refined definitions of substructures within the $4 \mathrm{WHO}$ entities of MB may be expected. The existence of such substructures might be a possible cause for the heterogeneity of the epicenter of the tumor in our study and the divergent reports of the typical location of WNTMBs in previous studies.

The children in our cohort were older than 4 years of age; this matches previous observations that WNT-MBs do not typically occur in early childhood. ${ }^{9}$ Most WNT-MBs in our cohort were histologically $\mathrm{CMBs}$, but there were single other histologic types (large-cell/anaplastic MB, $n=2$ ) as well. The fact that WNT-MBs are not exclusively CMBs has been reported previously. ${ }^{9,10,13}$ Our cohort contained 1 tumor corresponding to an LCMB (according to the WHO classification of 2007). This is in accordance with Ellison et $\mathrm{al}^{9}$ and Kool et $\mathrm{al}^{10}$ reporting 2\% LCMBs among their group of WNT-MBs. With a mean tumor volume of $15 \mathrm{~cm}^{3}$, LCMBs have been described as significantly smaller than other histologic types of $\mathrm{MB}^{22}$ Most interesting, $10 \mathrm{CMBs}$ in our cohort had a tumor size of $>15 \mathrm{~cm}^{3}$, and the only LCMB showed double that size. In our cohort, $11.5 \%$ of patients showed a macroscopic leptomeningeal dissemination at diagnosis. This percentage is similar to the numbers reported in studies on neuropathology but higher than in previous radiologic studies. ${ }^{9,13,15,30}$ We found a female predominance, which is contrary to that in the cohort of Patay et al. ${ }^{14}$ The female predominance in our cohort should be considered with caution due to the small cohort size in comparison with publications that have recorded the demographic data of significantly more WNT-MBs and found no predominance of male or female. , $^{9,10,26}$

MBs are tumors of high cellularity for which a lower signal on T2WI can be expected. In our cohort, WNT-MBs were primarily iso- to hypointense on T2WI. The comparatively low T2 signal is useful to differentiate MBs from pilocytic astrocytomas and, to a lesser extent, also from ependymomas. Inhomogeneous signal on T2WI and the moderate-to-strong contrast enhancement do not seem to be specific criteria to separate WNT-MBs from other highly cellular tumors, for example, atypical teratoid/rhabdoid tumors. So far, the literature has been heterogeneous with regard to intratumoral blood-degradation products in MBs. Patay et al ${ }^{14}$ reported blood-degradation products in only $31.25 \%$, whereas Reisinger et $\mathrm{al}^{31}$ found substantial intratumoral hemorrhage in 54\% of assessed tumors. Still another group (Perreault et $\mathrm{al}^{12}$ ) did not find any subgroup-specific features on iron-sensitive images. However, as one of the major findings in our study, a high proportion of tumors showed large areas of methemoglobin or hemosiderin (60.5\%) and cysts containing blood-degradation products with blood-fluid levels.

\section{Limitations}

We acknowledge as a limitation that in this work, we only examined the WNT subgroup for imaging characteristics and, thus, did not directly compare the results with those of other MB subgroups. Due to the multicenter principle, we assessed imaging data from MR imaging scanners at different magnetic field strengths and sequence techniques. Thus, heterogeneity of imaging data could not be completely controlled.

\section{CONCLUSIONS}

WNT-MBs are found predominately in the fourth ventricle. However, a certain laterality can be noted by their hypothetic point of origin and possible growth characteristics. However, hemispherically positioned WNT-MBs and leptomeningeal dissemination can occur and may not be used as a criterion to exclude WNT-MB. Dense intratumoral blood-degradation products and cysts with blood contents are frequently found and might help to differentiate WNT-MBs from other MB subtypes.

\section{ACKNOWLEDGMENTS}

We thank Dr D. Engel for her support in manuscript correction.

Disclosures: Annika Stock-RELATED: Grant: German Children's Cancer Foundation.* Torsten Pietsch-RELATED: Grant: German Children's Cancer Foundation, Comments: grant money for reference pathologic evaluation and retrospective reclassification according to revised World Health Organization classification.* Steven C. Clifford-RELATED: Grant: Cancer Research UK, Comments: research grant.* Stefan Rutkowski-RELATED: Grant: German Children's Cancer Foundation.* Brigitte Bison—RELATED: Grant: German Children's Cancer Foundation, Comments: The Neuroradiological Reference Center, as part of the HIT-network, is supported by the German Children's Cancer Foundation*; Support for Travel to Meetings for the Study or Other Purposes: Deutsche Kinderkrebsstiftung, Comments: Travel Support is to The Reference Center for the German Brain Tumor Studies by Deutsche Kinderkrebsstiftung.* Mirko PhamRELATED: Grant: German Children's Cancer Foundation*; UNRELATED: Grants/ Grants Pending: Deutsche Forschungsgemeinschaft (CRC TR 240 B02, CRC 1158/1 A03, CRC 1158/2 A10)*; Payment for lectures/travel expenses from Bayer AG and Merck Serono $\mathrm{GmbH}$, Comments: speaker honoraria.* Monika Warmuth-MetzRELATED: Grant: German Children's Cancer Foundation, Comments: The reference center is only possible with the permanent financial support of the German Children's Cancer Foundation, which is the association of parents of children with cancer*; UNRELATED: Grants/Grants Pending: German Children's Cancer Foundation, Comments: The reference center is only possible with the permanent financial support of the German Children's Cancer Foundation, which is the association of parents of children with cancer.* Martin Mynarek-RELATED: Grant: German Children's Cancer Foundation*; UNRELATED: Grants/Grants Pending: Riemser Pharma, Greifswald, Germany, Comments: for activities outside of this work.* *Money paid to the institution/personal.

\section{REFERENCES}

1. Louis DN, Ohgaki H, Wiestler OD, et al, eds. WHO Classification of Tumours of the Central Nervous System, 4th ed. Geneva: IARC Press; 2016

2. Louis DN, Wiestler OD, Cavenee WK, et al. WHO Classification of Tumors of the Central Nervous System. Geneva: IARC Press; 2007

3. Koch A, Waha A, Tonn JC, et al. Somatic mutations of WNT/wingless signaling pathway components in primitive neuroectodermal tumors. Int J Cancer 2001;93:445-49 CrossRef Medline

4. Ellison DW, Onilude OE, Lindsey JC, et al. Beta-catenin status predicts a favorable outcome in childhood medulloblastoma: the United Kingdom Children's Cancer Study Group Brain Tumour Committee. J Clin Oncol 2005;23:7951957 CrossRef Medline

5. Waszak SM, Northcott PA, Buchhalter I, et al. Spectrum and prevalence of genetic predisposition in medulloblastoma: a retrospective genetic study and prospective validation in a clinical trial cohort. Lancet Oncol 2018;19:785-98 CrossRef Medline 
6. Baeza N, Masuoka J, Kleihues P, et al. AXIN1 mutations but not deletions in cerebellar medulloblastomas. Oncogene 2003;22:632-36 CrossRef Medline

7. Dahmen RP, Koch A, Denkhaus D, et al. Deletions of AXIN1, a component of the WNT/wingless pathway, in sporadic medulloblastomas. Cancer Res 2001;61:7039-43 Medline

8. Koch A, Hrychyk A, Hartmann W, et al. Mutations of the Wnt antagonist AXIN2 (Conductin) result in TCF-dependent transcription in medulloblastomas. Int J Cancer 2007;121:284-91 CrossRef Medline

9. Ellison DW, Dalton J, Kocak M, et al. Medulloblastoma: clinicopathological correlates of SHH, WNT, and non-SHH/WNT molecular subgroups. Acta Neuropathol 2011;121:381-96 CrossRef Medline

10. Kool M, Korshunov A, Remke M, et al. Molecular subgroups of medulloblastoma. An international meta-analysis of transcriptome, genetic aberrations, and clinical data of WNT, SHH, group 3, and group 4 medulloblastomas. Acta Neuropathol 2012;123:47384 CrossRef Medline

11. Goschzik T, Schwalbe EC, Hicks D, et al. Prognostic effect of whole chromosomal aberration signatures in standard-risk, non-WNT/ non-SHH medulloblastoma: a retrospective, molecular analysis of the HIT-SIOP PNET 4 trial. Lancet Oncol 2018;19:1602-16 CrossRef Medline

12. Perreault $\mathrm{S}$, Ramaswamy V, Achrol AS, et al. MRI surrogates for molecular subgroups of medulloblastoma. AJNR Am J Neuroradiol 2014;35:1263-69 CrossRef Medline

13. Mata-Mbemba D, Zapotocky M, Laughlin S, et al. MRI characteristics of primary tumors and metastatic lesions in molecular subgroups of pediatric medulloblastoma. a single-center study. AJNR Am J Neuroradiol 2018;39:949-55 CrossRef Medline

14. Patay Z, DeSain LA, Hwang SN, et al. MR imaging characteristics of wingless-type-subgroup pediatric medulloblastoma. AJNR Am J Neuroradiol 2015;36:2386-93 CrossRef Medline

15. Dasgupta A, Gupta T, Pungavkar S, et al. Nomograms based on preoperative multiparametric magnetic resonance imaging for prediction of molecular subgrouping in medulloblastoma: results from a radiogenomics study of 111 patients. Neuro Oncol 2019;21:115-24 CrossRef Medline

16. Pietsch T, Haberler C. Update on the integrated histopathological and genetic classification of medulloblastoma: a practical diagnostic guideline. Clin Neuropathol 2016;35:344-52 CrossRef Medline

17. Goschzik T, Zur Mühlen A, Kristiansen G, et al. Molecular stratification of medulloblastoma: comparison of histological and genetic methods to detect Wnt activated tumours. Neuropathol Appl Neurobiol 2015;41:135-44 CrossRef Medline
18. Pietsch T, Schmidt R, Remke M, et al. Prognostic significance of clinical, histopathological, and molecular characteristics of medulloblastomas in the prospective HIT2000 multicenter clinical trial cohort. Acta Neuropathol 2014;128:137-49 CrossRef Medline

19. Capper D, Jones DT, Sill M, et al. DNA methylation-based classification of central nervous system tumours. Nature 2018;555:469-74 CrossRef Medline

20. Schwalbe EC, Hicks D, Rafiee G, et al. Minimal methylation classifier (MIMIC). A novel method for derivation and rapid diagnostic detection of disease-associated DNA methylation signatures. Sci Rep 2017;7:13421 CrossRef Medline

21. Chang $\mathrm{CH}$, Housepian EM, Herbert C. An operative staging system and a megavoltage radiotherapeutic technic for cerebellar medulloblastomas. Radiology 1969;93:1351-59 CrossRef Medline

22. Warmuth-Metz M. Imaging and Diagnosis in Pediatric Brain Tumor Studies. Cham: Springer-Verlag International Publishing; 2017:8-12

23. Gibson P, Tong Y, Robinson G, et al. Subtypes of medulloblastoma have distinct developmental origins. Nature 2010;468:1095-99 CrossRef Medline

24. Łastowska M, Jurkiewicz E, Trubicka J, et al. Contrast enhancement pattern predicts poor survival for patients with non-WNT/SHH medulloblastoma tumours. J Neurooncol 2015;123:65-73 CrossRef Medline

25. Teo WY, Shen J, Su JM, et al. Implications of tumor location on subtypes of medulloblastoma. Pediatr Blood Cancer 2013;60:140810 CrossRef Medline

26. Taylor MD, Northcott PA, Korshunov A, et al. Molecular subgroups of medulloblastoma: the current consensus. Acta Neuropathol 2012;123:465-72 CrossRef Medline

27. Northcott PA, Buchhalter I, Morrissy AS, et al. The whole-genome landscape of medulloblastoma subtypes. Nature 2017;547:311-17 CrossRef Medline

28. Schwalbe EC, Lindsey JC, Nakjang S, et al. Novel molecular subgroups for clinical classification and outcome prediction in childhood medulloblastoma: a cohort study. Lancet Oncol 2017;18:95871 CrossRef Medline

29. Cavalli FM, Remke M, Rampasek L, et al. Intertumoral heterogeneity within medulloblastoma subgroups. Cancer Cell 2017;31:737-54 e6 CrossRef Medline

30. Zapotocky M, Mata-Mbemba D, Sumerauer D, et al. Differential patterns of metastatic dissemination across medulloblastoma subgroups. J Neurosurg Pediatr 2018;21:145-52 CrossRef Medline

31. Reisinger D, Gojo J, Kasprian G, et al. Predisposition of wingless subgroup medulloblastoma for primary tumor hemorrhage. Neurosurgery 2019 May 7. [Epub ahead of print] CrossRef Medline 